\title{
Noiseless Attenuation for Continuous-Variable Quantum Key Distribution over Ground-Satellite Uplink
}

\author{
Shengjie Xu ${ }^{1,2,+}$, Yin $\mathrm{Li}^{1,+}$, Yijun Wang ${ }^{1}$, Yun Mao ${ }^{1, *}$, Zhiyue Zuo ${ }^{1, *}$, Xinchao Ruan ${ }^{1}$ and Ying Guo ${ }^{1} \mathbb{D}$ \\ 1 School of Automation, Central South University, Changsha 410083, China; 206166@csu.edu.cn (S.X.); \\ liyin@csu.edu.cn (Y.L.); xxywyj@csu.edu.cn (Y.W.); rxc1126@csu.edu.cn (X.R.); yingguo@csu.edu.cn (Y.G.) \\ 2 School of Economics and Mangement, Beihua University, Jilin City 132013, China \\ * Correspondence: maoyun3106@csu.edu.cn (Y.M.); zuozuo@csu.edu.cn (Z.Z.) \\ + These authors contributed equally to this work.
}

Citation: Xu, S.; Li, Y.; Wang, Y.; Mao,

Y.; Zuo, Z.; Ruan, X.; Guo, Y.

Noiseless Attenuation for

Continuous-Variable Quantum Key Distribution over Ground-Satellite

Uplink. Appl. Sci. 2021, 11, 11289.

https://doi.org/10.3390/

app112311289

Academic Editor: Nuno Silva

Received: 28 October 2021

Accepted: 25 November 2021

Published: 29 November 2021

Publisher's Note: MDPI stays neutral with regard to jurisdictional claims in published maps and institutional affiliations.

Copyright: (c) 2021 by the authors. Licensee MDPI, Basel, Switzerland. This article is an open access article distributed under the terms and conditions of the Creative Commons Attribution (CC BY) license (https:// creativecommons.org/licenses/by/ $4.0 /)$.

\begin{abstract}
Satellite-based quantum key distribution (QKD) has lately received considerable attention due to its potential to establish a secure global network. Associated with its application is a turbulent atmosphere that sets a notable restriction to the transmission efficiency, which is especially challenging for ground-to-satellite uplink scenarios. Here, we propose a novel noiseless attenuation (NA) scheme involving a zero-photon catalysis operation for source preparation to improve the performance of continuous-variable (CV) QKD over uplink. Numerical analysis shows that the NA-based CV-QKD, under attenuation optimization, outperforms the traditional CV-QKD, which is embodied in extending the allowable zenith angle while improving the effective communication time. Attributing to characteristics of the attenuation optimization, we find that the NA-involved source preparation improves the security bound by relatively reducing the amount of information available to eavesdroppers. Taking the finite-size effect into account, we achieve a tighter bond of security, which is more practical compared with the asymptotic limit.
\end{abstract}

Keywords: quantum key distribution; continuous-variable; noiseless attenuation; uplink

\section{Introduction}

In recent decades, a number of quantum cryptography protocols have been successfully realized, including quantum teleportation (QT) [1,2], quantum key distribution (QKD) [3], quantum secret sharing [4-6], and quantum coding [7,8]. QKD, which mainly relies on Heisenberg's uncertainty principle, which provide us with an unconditional secure method for private information transmission over an insecure channel. Generally, it can be divided into two categories, referred to as discrete-variable (DV) QKD [9] and continuous-variable (CV) QKD [10]. In DV-QKD, the information is usually encoded in the polarization or time bin of single photons, using expensive and extreme cryo-cooling single-photon detectors [11]. Compared with DV-QKD, CV-QKD, which involves the coherent state, can achieve higher channel capacity by using homodyne or heterodyne detection, and has good compatibility with standard optical communications [12]. To date, CV-QKD has been successfully analyzed in fiber links both theoretical and experimental [13,14].

However, due to heavy losses in the practical fiber, the distance over which the coherent state of light can be transmitted is restricted within hundreds of kilometers. So far, the longest transmission distance of CV-QKD is only $202.81 \mathrm{~km}$ [3]. Since it is difficult to noiselessly amplify the quantum state at the receiver [15], exhibiting a global-scale network through a fiber link becomes a challenge. In the last few years, the research towards global-scale network has increased for a satellite-mediated scenario [16], where the channel losses are limited since the effective thickness of the atmosphere is only about $20 \mathrm{~km}$ [17]. The major works are being performed in downlink configuration, however, an uplink from ground to a quantum satellite is less explored because the atmospheric 
turbulence acts on early stages of optical signal transmission, leading to a larger diffractioninduced broadening and more serious beam wandering [18]. As has been pointed out, the secret key rate in uplink is less than one order of magnitude compared with the downlink scenario [19]. Despite the inferior performance compared with that of the downlink, the uplink CV-QKD is also of great value owing to the simple satellite structure, the variability of quantum sources, and the accessibility for maintenance repair [20,21].

To improve the performance of the uplink CV-QKD, we consider an enhanced source preparation scheme involving the noiseless attenuation (NA) operation [22]. The NA operation has been used for quantum key distribution, quantum teleportation, and other applications to reduce the detrimental effects of channel losses [23-25]. On the premise of the previous achievements, in this paper, we suggest an NA-based enhancement scheme for the Gaussian-modulated coherent-state protocol of uplink. Due to the limited space on the quantum satellite, the NA operation is applied to the ground station (the sender). We perform a suitable NA process by using a zero-photon catalysis (ZPC) operation, which happens to be a zero-energy-input operation that does not introduce the consumption of external energy [26]. We prove the security of the NA-based CV-QKD protocol against the Gaussian collective attack under both the asymptotic case and finite-size regime. Our results show that both the allowable zenith angle and effective communication time are improved compared with the traditional CV-QKD protocol. The research confirms the role of the NA operation on the uplink CV-QKD protocol and is conducive to making the CV-QKD protocol more practical.

The paper is organized as follows. In Section 2, we illustrate characteristics of the uplink CV-QKD system. In Section 3, we suggest the NA-based source preparation scheme, based on zero-photon catalysis, for improving the performance of the CV-QKD system. Section 4 shows the security of the NA-based protocol under both the asymptotic case and the finite-size regime. Finally, conclusions are drawn in Section 5. The motivation of this work is to improve the performance of the uplink CV-QKD to achieve a complete quantum network with more efficiency.

\section{Uplink CV-QKD}

For the convenience of security analysis, the uplink CV-QKD protocol is structured with the equivalent entanglement-based (EB) scheme [27]. As shown in Figure 1, Alice generates a two-mode squeezed vacuum (TMSV) state as an entangled resource, which can be represented by its covariance matrix

$$
\gamma_{A B}=\left[\begin{array}{cc}
V \Pi & \sqrt{V^{2}-1} \sigma_{z} \\
\sqrt{V^{2}-1} \sigma_{z} & V \Pi
\end{array}\right],
$$

where $\sigma_{z}=\operatorname{diag}(1,-1), \Pi=\operatorname{diag}(1,1)$ and $V=\left(1+\lambda^{2}\right) /\left(1-\lambda^{2}\right)$ with squeezing parameter $\lambda$. Then, Alice holds mode A for heterodyne detection, while sending another mode B to Bob over the insecure uplink, which is assumed to be controlled by a potential eavesdropper. Due to the random fading of the channel, the initial quantum state transfer to a mixture [28], whose composition depends on the random transmittance $T$ together with channel excess noise $\epsilon$. Here, we assume a fixed $\epsilon$, and describe the random $T$ by its probabilities distribution. Specifically, we divide the fading channel into $K$ stable subchannels of individuals, while each sub-channel has a fixed transmittance $\left\{T_{j}\right\}_{j \in\{1, \cdots, K\}}$ with corresponding probability $\left\{P_{j}\right\}_{j \in\{1, \cdots, K\}}$. Therefore, the arriving mixture shared by Alice and Bob is

$$
\gamma_{A B_{2}}=\left[\begin{array}{cc}
V \Pi & \langle\sqrt{T}\rangle \sqrt{V^{2}-1} \sigma_{z} \\
\langle\sqrt{T}\rangle \sqrt{V^{2}-1} \sigma_{z} & \langle T\rangle\left(V+\chi_{\text {line }}\right) \Pi
\end{array}\right]
$$


where $\langle T\rangle=\sum_{j=1}^{K} P_{j} T_{j},\langle\sqrt{T}\rangle=\sum_{j=1}^{K} P_{j} \sqrt{T}_{j}$, and $\chi_{\text {line }}=1 /\langle T\rangle-1+\epsilon$. Finally, by implementing quadrature measurement, classical information reconciliation, and privacy amplification, Alice and Bob can extract the secret keys.

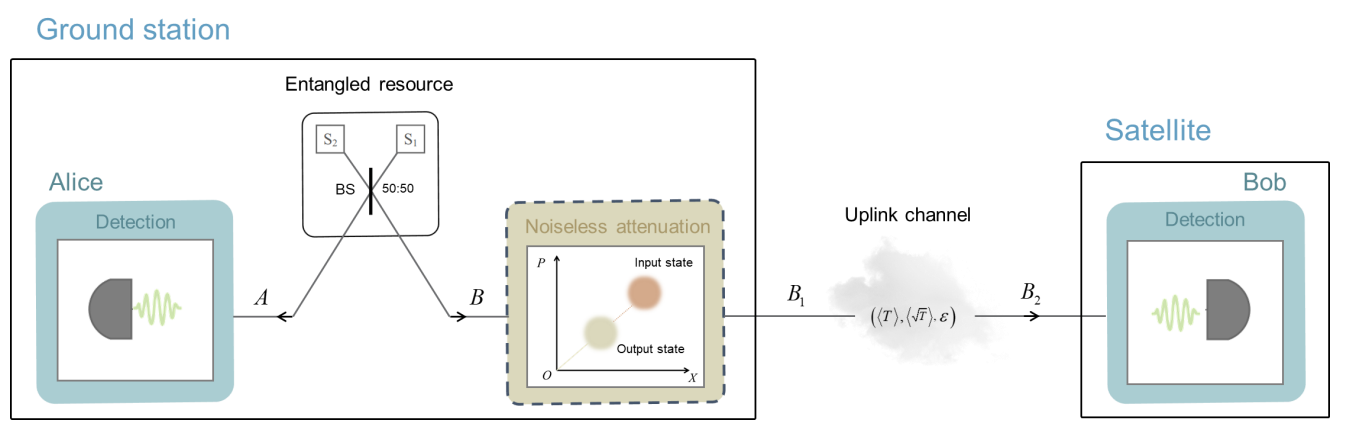

Figure 1. EB scheme of the NA-based CV-QKD in the uplink scenario. BS: beam splitter; $T$ : channel transmittance; $\epsilon$ : channel excess noise; $S_{1}, S_{2}$ : single-mode squeezed state.

As shown in Equation (2), the statistical parameters $\langle T\rangle$ and $\langle\sqrt{T}\rangle$ represents the fading effects of uplink on quantum state. To describe these parameters, we apply the elliptic-beam model $[29,30]$, which provides accurate agreement with the experimental data under weak and strong turbulence. In this model, the receiving beam is assumed to be an elliptic deformation, which can be expressed by a random vector

$$
v=\left(x_{0}, y_{0}, W_{1}, W_{2}, \varphi\right),
$$

where $\left(x_{0}, y_{0}\right)$ denotes the beam-centroid coordinates at telescope plane, $W_{1}$ and $W_{2}$ are the semi-axis of arriving elliptic beam, and $\varphi$ is the orientation angle between semi-axis $W_{1}$ and the $x$ axis. Based on the vector $v$, the probability distribution of transmittance (PDT) is given by

$$
P(T)=\frac{2}{\pi} \int_{\mathbb{R}^{4}} \mathrm{~d}^{4} v \int_{0}^{\frac{\pi}{2}} \mathrm{~d} \varphi \rho_{G}(v ; \mu, \Sigma) \delta(T-T(v)),
$$

where $T(v)$ defines the relationship between $T$ and $v$, and $\rho_{G}(v ; \mu, \Sigma)$ is a Gaussian probability density of $v$ with the mean value $\mu$ and the covariance matrix $\Sigma$. The elements of $\mu$ and $\Sigma$ in the uplink case are listed in Appendix A. Based on $\mu$ and $\Sigma$, we can derive the PDT of the uplink channel through the Monte Carlo method [29], and then obtain the statistical parameters of $T$. Note that the simulation parameters of the work (as shown in Table 1) are the same as our previous work so that the numerical analysis of transmittance vs. zenith angle can refer to [21].

Subsequently, we demonstrate the performance of the uplink CV-QKD protocol. We consider the asymptotic regime and assume an ideal homodyne detection. The information reconciliation is the reverse reconciliation, since it is more robust against channel attenuation [31]. The secret key rate can be derived from Equation (2) with the support of Gaussian state extremality theorem [27], which is given by

$$
K_{A}(\langle T\rangle,\langle\sqrt{T}\rangle, \epsilon)=\beta_{r} I_{A B}-\chi_{E},
$$

where $\beta_{r}$ denotes the reconciliation efficiency, $I_{A B}$ is the Shanon mutual information, and $\chi_{E}$ represents the Holevo bound. The calculations of $I_{A B}$ and $\chi_{E}$ are shown in Appendix B. Moreover, the angle-of-arrival fluctuation caused by wavefront distortion will introduce a coupling problem between spatial beam and fiber on receiver detection [32]. The relationship between the fiber coupling efficiency $\varsigma$ and angle-of-arrival $\beta_{a}$ can be expressed as [33]

$$
\varsigma=\int_{-0.5 d_{\text {cor }}}^{0.5 d_{\text {cor }}} \frac{1}{\sqrt{2 \pi\left\langle\beta_{a}^{2}\right\rangle} f} \exp \left(\frac{-l^{2}}{2 f^{2}\left\langle\beta_{a}^{2}\right\rangle}\right) \mathrm{d} l,
$$


where $f$ denotes the focal length, $d_{\text {cor }}$ is the diameter of the fiber. $\left\langle\beta_{a}^{2}\right\rangle$ represents the variance of $\beta_{a}$, which is related to refraction index structure parameter $C_{n}^{2}$. Details of $\left\langle\beta_{a}^{2}\right\rangle$ in uplink case refer to Appendix C. Hence, the secret key rate in Equation (5) should be modified to

$$
K_{A}(\langle T\rangle,\langle\sqrt{T}\rangle, \epsilon)=\varsigma \times\left(\beta_{r} I_{A B}-\chi_{E}\right) .
$$

Table 1. The simulation parameters.

\begin{tabular}{cccc}
\hline Variable & Value & Description & Reference \\
\hline$\zeta$ & $0-60^{\circ}$ & Zenith angle & \\
$a$ & $1 \mathrm{~m}$ & Receiver telescope radius & {$[21]$} \\
$\mathrm{f} / 2 \mathrm{a}$ & 1 & Focal ratio & {$[33]$} \\
$d_{\text {cor }}$ & $10 \mu \mathrm{m}$ & Fiber core diameter & {$[33]$} \\
$W_{0}$ & $80 \mathrm{~mm}$ & Intial beam radius & {$[33]$} \\
$V-1$ & $4 \mathrm{SNU}$ & Modulation variance & {$[34]$} \\
$\lambda_{0}$ & $800 \mathrm{~nm}$ & Wavelength & {$[21]$} \\
$\beta_{r}$ & $95 \%$ & Reconciliation efficiency & {$[21]$} \\
$\epsilon$ & $0.01 \mathrm{SNU}$ & Excess noise & {$[35]$} \\
$C_{n}^{2}$ & $1.12 \times 10^{-16} \mathrm{~m}^{-2 / 3}$ & Refraction index structure parameter & {$[17]$} \\
$n_{0}$ & $0.61 \mathrm{~m}^{-3}$ & Mean number of scatterer particles & {$[17]$} \\
\hline
\end{tabular}

The secret key rates $K_{A}$ as a function of the zenith angle $\zeta$ for the given satellite altitude $H$ is shown in Figure 2, where the simulation parameters refer to Table 1 in Section 4. In addition, the performance of the downlink scenario, analyzing with the same parameters, is also plotted for comparison. Numerical analysis reveals the favorable of downlink for communication, while illustrating the urgency of an enhancement scheme for the uplink case. Note that the maximum secret key rate $\left(\zeta=0^{\circ}\right)$ of the downlink at $100 \mathrm{~km}$ is lower than that of the uplink because the fiber coupling problem is more severe in the downlink scenario [21].

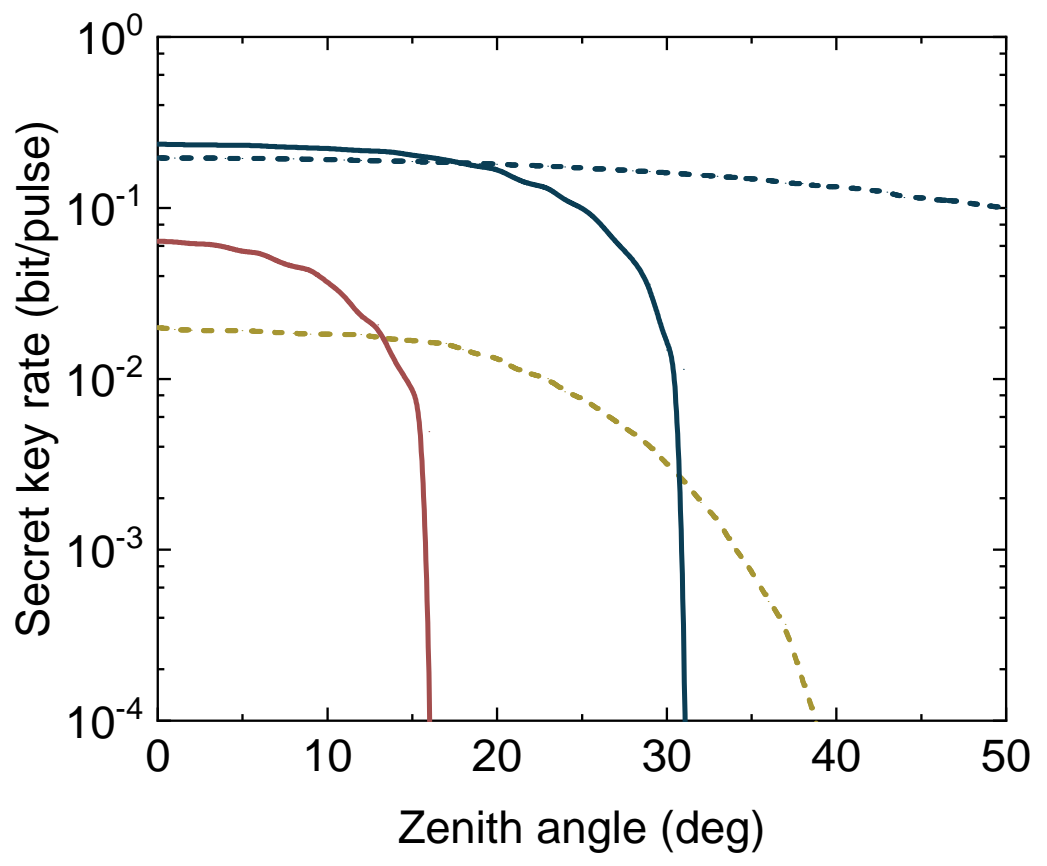

Figure 2. Secret key rate vs. zenith angle for different scenarios: downlink (dashed line) and uplink (solid line). The blue, red, and yellow lines represent the satellite altitude of $100 \mathrm{~km}, 120 \mathrm{~km}$, and $800 \mathrm{~km}$, respectively. 


\section{Modified Protocol with Noiseless Attenuation}

Due to the limited size of the quantum satellite, an enhanced source preparation scheme at the ground station becomes a feasible solution. Motivated by characteristics of the source preparation [23-25], we apply a suitable NA operation before transmission for performance improvement (see Figure 1). In this section, the NA-based source preparation is realized via the ZPC operation, which can be implemented by mixing zero photon at an asymmetrical beam splitter (ABS) and then post-selecting the ABS output by conditional detection of zero photon (see Figure 3). For the TMSV state, the catalyzed mode (mode B) is sent to one of the input ports of ABS with transmittance $T_{A}$, while a zero-photon ancillary Fock state incidents on another port for interference. In an ideal case, the auxiliary zero-photon state will not be destroyed at all and the photon number resolving detector (PNRD) at the corresponding output ports can register zero photon. Therefore, the ZPCinvolved attenuation happens to be a zero-energy-input attenuation that does not cause the consumption of external energy. Mathematically, the ZPC process can be regarded as an equivalent operation $\hat{O}_{0}$ given by

$$
\hat{O}_{0}=\frac{\partial^{m}}{m ! \partial \chi^{m}}\left\{\frac{T_{A}^{m}}{1-\chi}\left(\frac{\sqrt{T}_{A}-\chi / \sqrt{T}_{A}}{1-\chi}\right)^{b^{\dagger} b}\right\}_{m=0, \chi=0},
$$

where $b^{\dagger}(b)$ denotes the addition (annihilation) photon operation of input mode, $m$ is the number of photons detected by PNRD when the ZPC process is successful. Obviously, $m=0$ in the ZPC process as the description above.

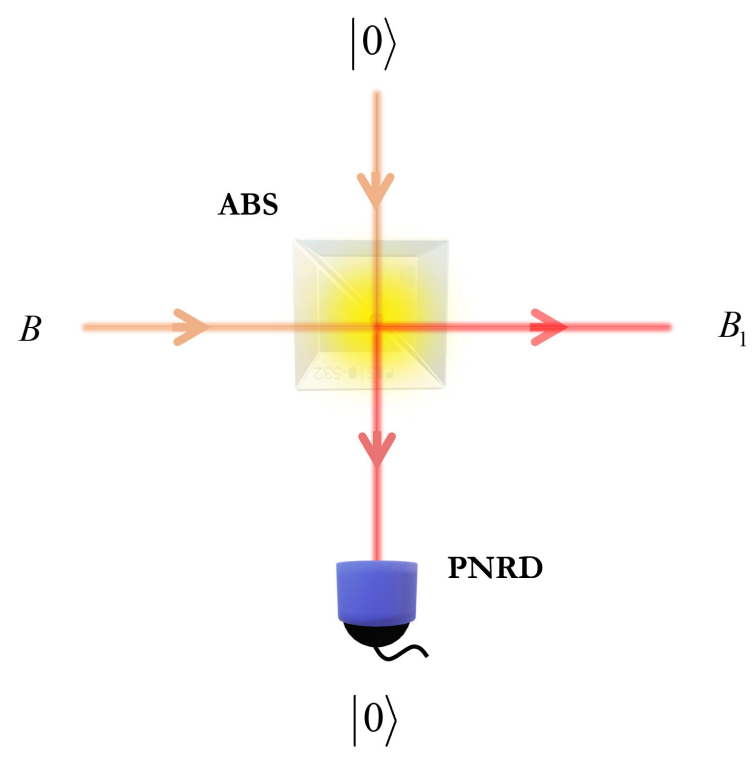

Figure 3. The ZPC operation for the NA-based source preparation. ABS: asymmetrical beam splitter. PNRD: photon number resolving detector.

When Alice performs heterodyne detection on mode $\mathrm{A}$, the transmission mode $\mathrm{B}$ will project to a coherent state [27]. It means that the ZPC operation on TMSV is equivalent to that acts on a coherent state, which can be written as

$$
\hat{O}_{0}|\alpha\rangle=\left(\sqrt{T_{A}}\right)^{b^{\dagger} b}|\alpha\rangle=\left|\sqrt{T_{A}} \alpha\right\rangle,
$$


where $|\alpha\rangle$ denotes the coherent state. We find that the ZPC operation happens to be an NA process with attenuation coefficient $g=\sqrt{T_{A}}$. In addition, the ZPC operation acting on a coherent state is a probability process with success probability

$$
P=\frac{2}{1+T_{A}+R V}
$$

where $R=1-T_{A}$. Figure 4 shows the relationship between ABS transmittance $T_{A}$ and attenuation coefficient $g$ with the corresponding success probability $P$. We find that both attenuation coefficient and success probability saw an upward trend with the increasing transmittance.

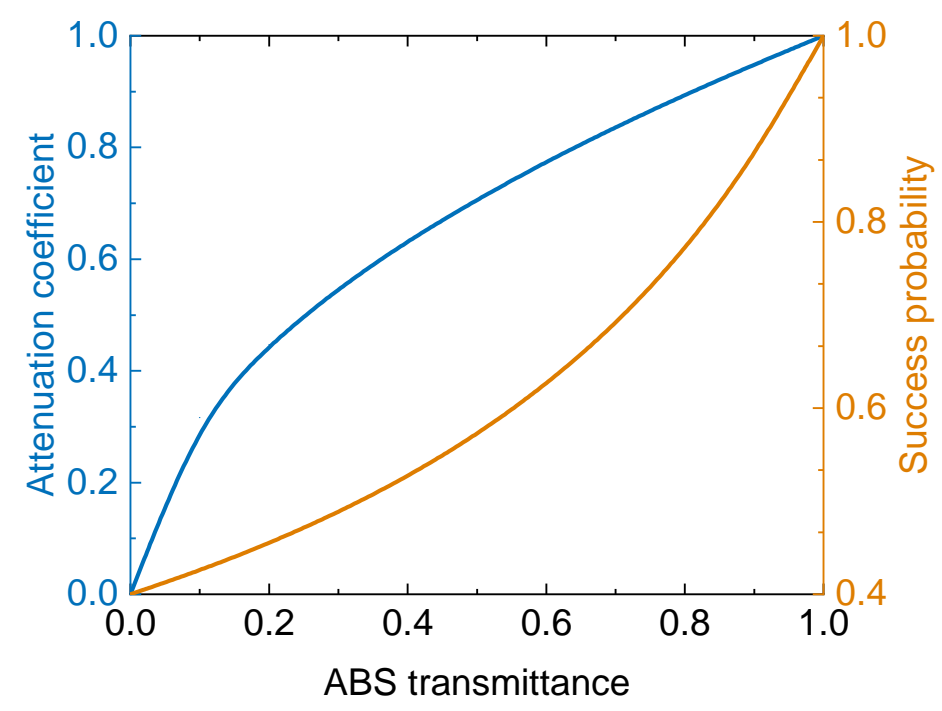

Figure 4. The attenuation coefficient vs. transmittance and the corresponding success probability.

We note that the covariance matrix of the ZPC-involved attenuation state can be derived by its characteristic function (CF), as shown in Appendix D. The form of the covariance matrix can be expressed as

$$
\gamma_{A B_{1}}=\left[\begin{array}{cc}
X \Pi & Y \sigma_{z} \\
Y \sigma_{z} & X \Pi
\end{array}\right]
$$

where the factors $\mathrm{X}$ and $\mathrm{Y}$ are listed as follows

$$
\begin{aligned}
& X=\frac{2 V-R V+R}{1+T_{A}+R V}, \\
& Y=\frac{2 \sqrt{T_{A}\left(V^{2}-1\right)}}{1+T_{A}+R V} .
\end{aligned}
$$

Then, the attenuation state transfers the uplink channel for quadrature measurement. The final covariance matrix of arriving attenuation state is given by

$$
\gamma_{A B_{2}}=\left[\begin{array}{cc}
X \Pi & \langle\sqrt{T}\rangle Y \sigma_{z} \\
\langle\sqrt{T}\rangle Y \sigma_{z} & \langle T\rangle\left(X+\chi_{\text {line }}\right) \Pi
\end{array}\right] .
$$

\section{Performance Analysis}

In this section, we perform the security analysis of the NA-based uplink CV-QKD protocol for the asymptotic case and the finite-size regime. Since the ZPC operation belongs to a kind of Gaussian operation (see Equation (9)), we can derive the secret key rate via the conventional method of the Gaussian protocol. The simulation parameters are shown in Table 1 . We consider a collimated Gaussian beam with an initial radius $W_{0}$ transmitted 
onto a telescope with radius $a$, and illustrate the results of clear night-time. Note that CV protocol has the potential to reach an all-day communication [36,37], and the performance of the day-time situation can be derived by updating the atmospheric parameters, i.e., refraction index structure parameter $C_{n}^{2}$, and the mean number of scatterer particles $n_{0}$.

\subsection{Asymptotic Analysis}

As shown in Figure 5a, it demonstrates the secret key rate $K_{A}$ as a function of zenith angle $\zeta$ for various satellite altitudes. The attenuation coefficient $g$ is optimal for each zenith angle. In addition, the single-photon catalysis (SPC) protocol, which can be considered as another noiseless attenuation operation [34], is also plotted for comparison. We find that the NA-based scheme improves performance. The allowable zenith angle is improved by $6^{\circ}$ and $8^{\circ}$ at $H=100 \mathrm{~km}$ and $H=120 \mathrm{~km}$, respectively. Note that the satellite-mediated CVQKD system has a strong dependence on the instantaneous position between the moving satellite and the ground station. The effective time of a communication cycle (satellite orbits the earth) is limited to the period when the satellite passes above the ground station. Therefore, a larger allowable zenith angle ensures a longer effective communication time so, as a consequence, more total secret keys. Figure $5 \mathrm{~b}$ reveals that the NA operation increases the effective time. The calculation is based on Newton's second law with the earth mass $M=5.965 \times 10^{24} \mathrm{~kg}$ and the earth radius $r_{0}=6371 \mathrm{~km}$.

(a)

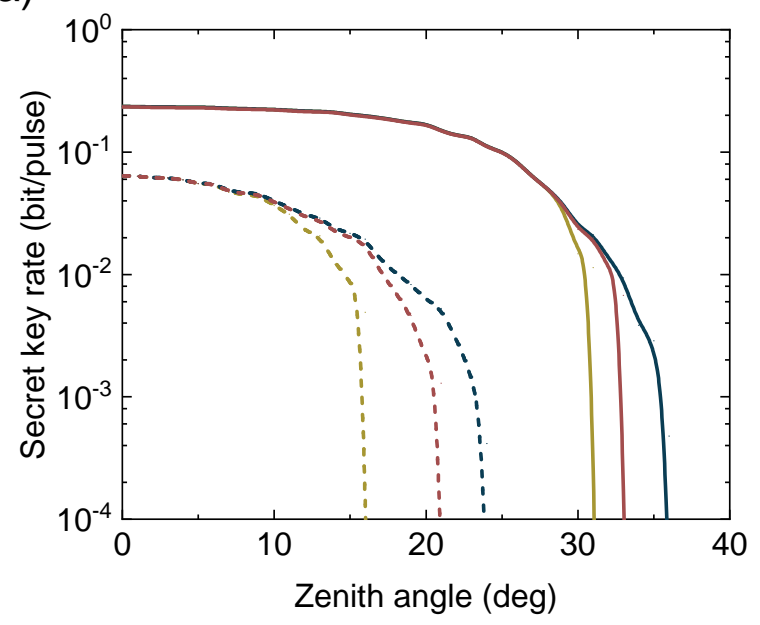

(b)

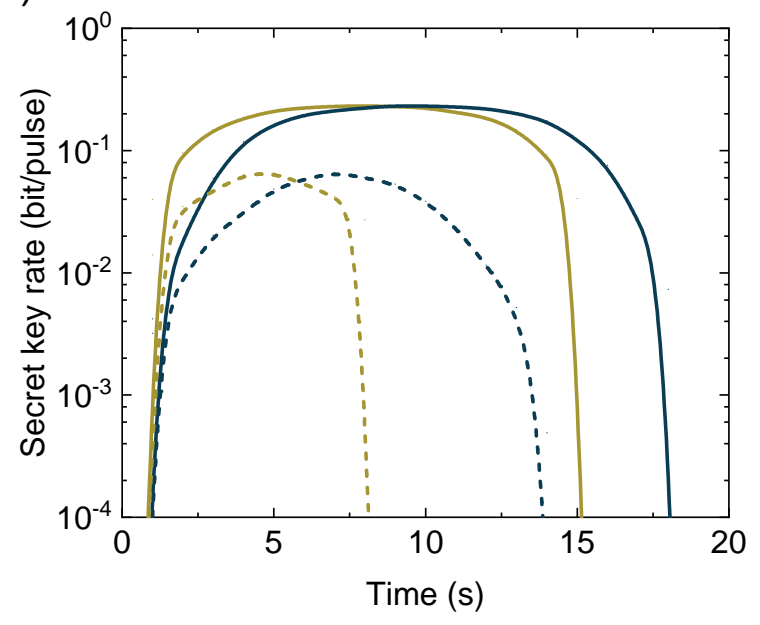

Figure 5. Secret key rate as a function of (a) zenith angle and (b) communication time during a single communication cycle. The blue, red, and yellow lines represent the case of ZPC-based protocol, SPC-based protocol, and coherent-state protocol, respectively. Solid lines and dashed lines represent two situations using the satellite altitude $H=100 \mathrm{~km}$ and $H=120 \mathrm{~km}$, respectively. 
Another observation in Figure 5 is that, at a small zenith angle, the bound of the secret key rate of the NA-based CV-QKD protocol coincident with that of the coherentstate protocol. As the zenith angle increases, so does the strength of turbulence, the improvement caused by the NA operation starts to appear. This phenomenon can be attributed to the optimization of attenuation coefficient. Figure 6 illustrates the optimal attenuation coefficient $g$ varying with the zenith angle $\zeta$ for various satellite altitudes. The optimal attenuation coefficient equals 1 for the case of small zenith angle $\left(\zeta<3^{\circ}\right.$ and $\zeta<26^{\circ}$ for $100 \mathrm{~km}$ and $120 \mathrm{~km}$, respectively), and then shows a downward trend with the increasing zenith angle. In other words, the protocol without NA operation may perform better than the NA-based one under weak turbulence effects, while the NA-based protocol can reach higher secret key rate when the turbulence effects become stronger. Moreover, the NA operation may be required upon higher satellite altitude at $H=120 \mathrm{~km}$.

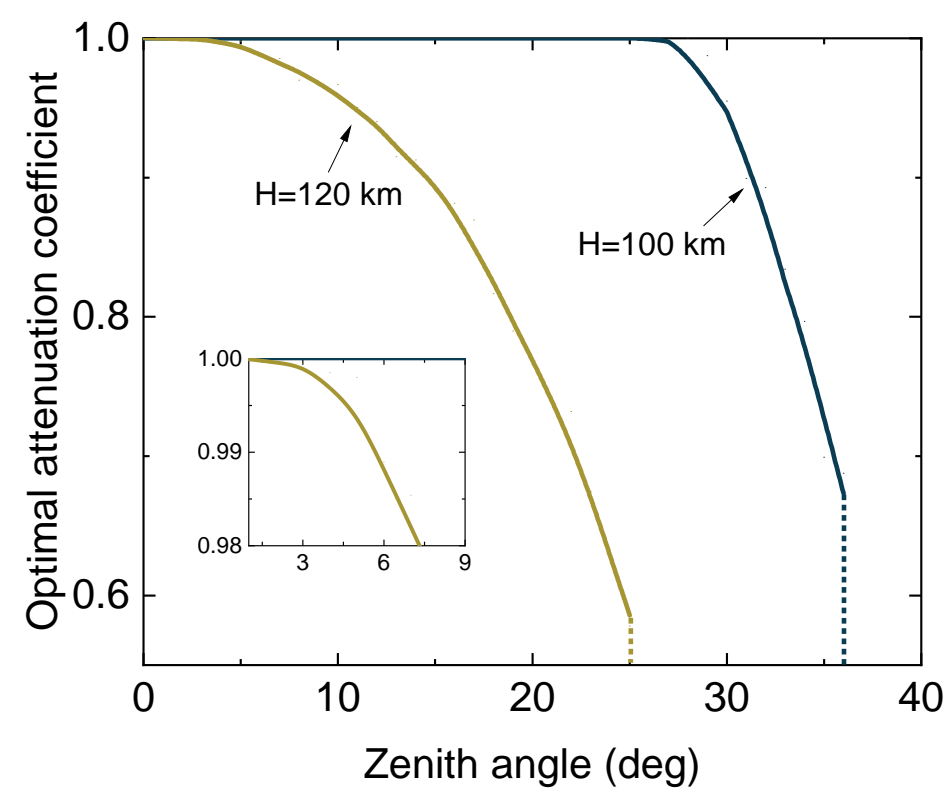

Figure 6. The optimal attenuation coefficient as a function of zenith angle for the satellite altitudes $H=100 \mathrm{~km}$ (blue line) and $H=120 \mathrm{~km}$ (yellow line).

In the following, we show the effect of the NA operation on the security of the CVQKD system. In Figure 7, we plot the reconciliation-stricken mutual information $\beta_{r} I_{A B}$ and Holevo bound $\chi_{E}$ as a function of attenuation coefficient $g$. Without loss of generality, we select $\zeta=0^{\circ}$ and $\zeta=32^{\circ}$ to represent small zenith angle case and large zenith angle case, respectively. In addition, the satellite altitude is set to $100 \mathrm{~km}$. We find that the use of the NA operation acts a negative effect on mutual information, but at the same time reduces the Holevo bound. For $\zeta=0^{\circ}$, the secret key rate, represented by the gap between the green line and purple line, keeps increasing as the growth attenuation coefficient. For a zenith angle of $32^{\circ}$, the secret key rate reaches the maximum at $g=0.834$, and then decreases with the growth of attenuation coefficient. In a word, the NA operation can improve the secret key rate under a large zenith angle by relatively reducing the amount of information available to Eve, which is accomplished by selecting an optimal attenuation coefficient. 
(a)

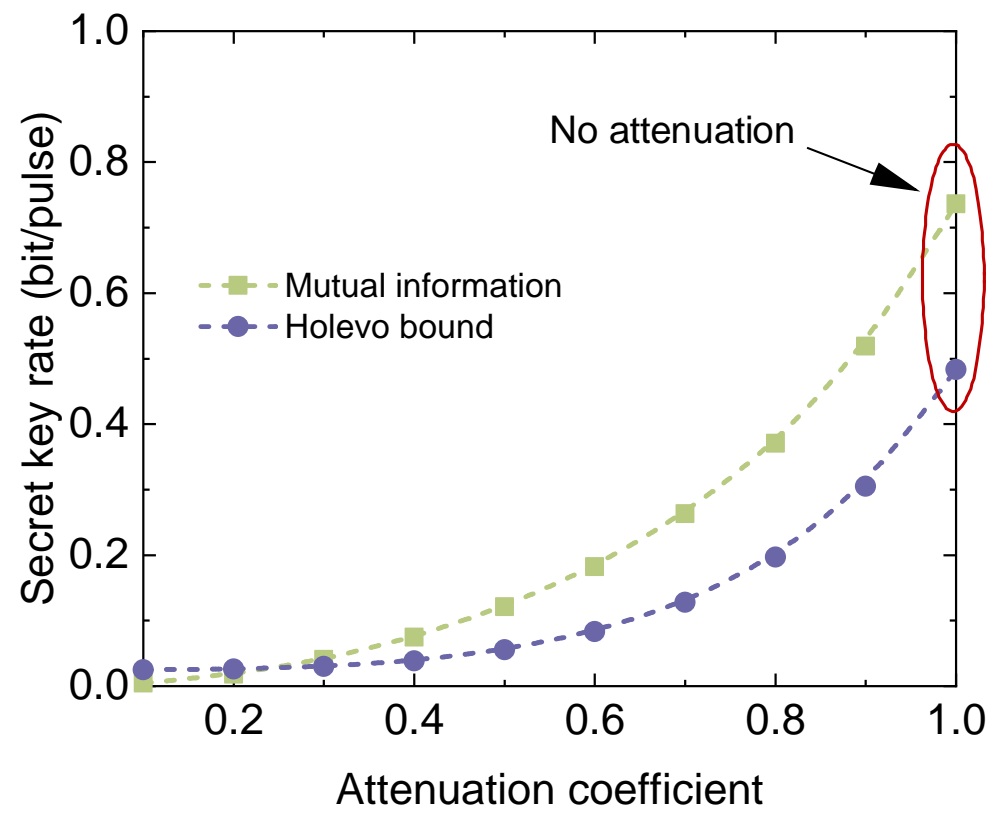

(b)

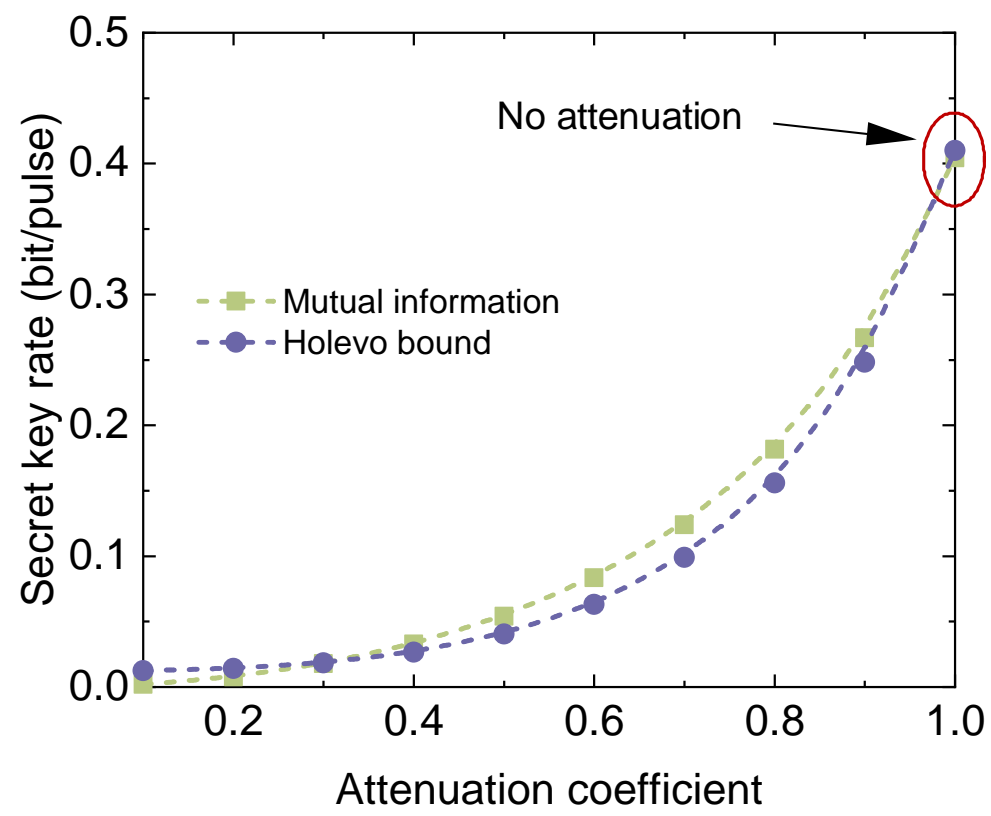

Figure 7. The reconciliation-stricken mutual information (green line) and Holevo bound (purple line) vs. attenuation coefficient for the zenith angles: (a) $\zeta=0^{\circ} ;(\mathbf{b}) \zeta=32^{\circ}$.

\subsection{Finite-Size Regime}

In the asymptotic case mentioned above, the secure analysis is performed with the premise that the length of the data is infinite, which is impossible in practice. Therefore, considering the finite-size effect in security analysis is necessary for practical implementations [38-40]. In the finite-size regime, we need to estimate the unknown parameters, including the channel transmittance and channel noise. In practice, these parameters are estimated using finite-sized data samples so that the secret key rate will be reduced. Although the finite-size effect in fiber link has been well studied, the analysis in free space is still a subject to be focused on since the channel fluctuation has a significant negative impact on the parameter estimation. To compensate for imprecise estimation, a suppression method, which divides the overall data into several clusters for fluctuation suppression, has 
been proposed [41]. However, it also increases the difficulty of the software and hardware at the receiver.

In this section, we consider the case that overall data are included for security analysis. With the overall data, the covariance matrix $\gamma_{A B_{2}}$ can be equivalently parameterized as originating from a stable link with fixed transmittance $\eta=\langle\sqrt{T}\rangle^{2}$, which is given by

$$
\gamma_{A B_{2}}=\left[\begin{array}{cc}
X \Pi & \sqrt{\eta} Y \sigma_{z} \\
\sqrt{\eta} Y \sigma_{z} & \eta(X-1)+\xi^{2}
\end{array}\right]
$$

where $\xi^{2}=\left(\langle T\rangle-\langle\sqrt{T}\rangle^{2}\right)(X-1)+1+\langle T\rangle \epsilon$. Following the rewritten covariance matrix above, the calculation of the secret key rate in finite-size regime can be expressed as

$$
K_{F}=\varsigma \times\left\{r\left[K_{A}\left(\eta_{\min }, \xi_{\max }^{2}\right)-\Delta(n)\right]\right\},
$$

where $r$ is the proportion of data used for secret key rate generation, $\Delta(n)$ is related to the security of the privacy amplification given by

$$
\Delta(n)=7 \sqrt{\frac{\log _{2}(2 / \bar{\epsilon})}{r \cdot N}}+\frac{2}{r \cdot N} \log _{2}\left(1 / \epsilon_{P A}\right),
$$

where $N$ represents the total exchanged signals, $\bar{\epsilon}$ is the smoothing parameter, and $\epsilon_{P A}$ is the failure probability of the privacy amplification procedure. Based on the derived results in [38], the value of $\eta_{\min }$ and $\xi_{\max }^{2}$ can be calculated by the maximum-likelihood estimators, which has the form

$$
\begin{aligned}
(\sqrt{\eta})_{\min } & \approx \sqrt{\eta}-Z_{\epsilon_{P E} / 2} \sqrt{\frac{1+\eta \xi}{N(1-r)(V-1)}}, \\
\xi_{\max }^{2} & \approx 1+\eta \xi+Z_{\epsilon_{P E} / 2} \frac{(1+\eta \xi) \sqrt{2}}{\sqrt{N(1-r)}}
\end{aligned}
$$

where $Z_{\epsilon_{P E} / 2}=\sqrt{2} \operatorname{erf}^{-1}\left(1-\epsilon_{P E}\right)$ with the failing probability of parameter estimation $\epsilon_{P E}$ and inverse error function $\operatorname{erf}^{-1}$. Here, erf represents the error function defined as

$$
\operatorname{erf}=\frac{2}{\sqrt{\pi}} \int_{0}^{x} e^{-t^{2}} \mathrm{~d} t
$$

Figure 8 shows the secret key rates $K_{F}$ as a function of the zenith angle $\zeta$ for various satellite altitudes, where the attenuation coefficient is optimal for each zenith angle. The parameters $\bar{\epsilon}, \epsilon_{P E}$, and $\epsilon_{P A}$ are $10^{-10}$, and half of the signals are used for parameter estimation $(r=0.5)$. We find that the fewer signals exchanged, the more pronounced of finite-size effect. When the number of the exchanged signals is $10^{6}$, the performance shows a big gap compared with other scenarios, especially in the case of $H=120 \mathrm{~km}$ (no secret key rate in that case). As the block length is increased, more signals can be used for parameter estimation and key extraction, so as a consequence, the secret key rate becomes closer to the asymptotic scenario. 
(a)

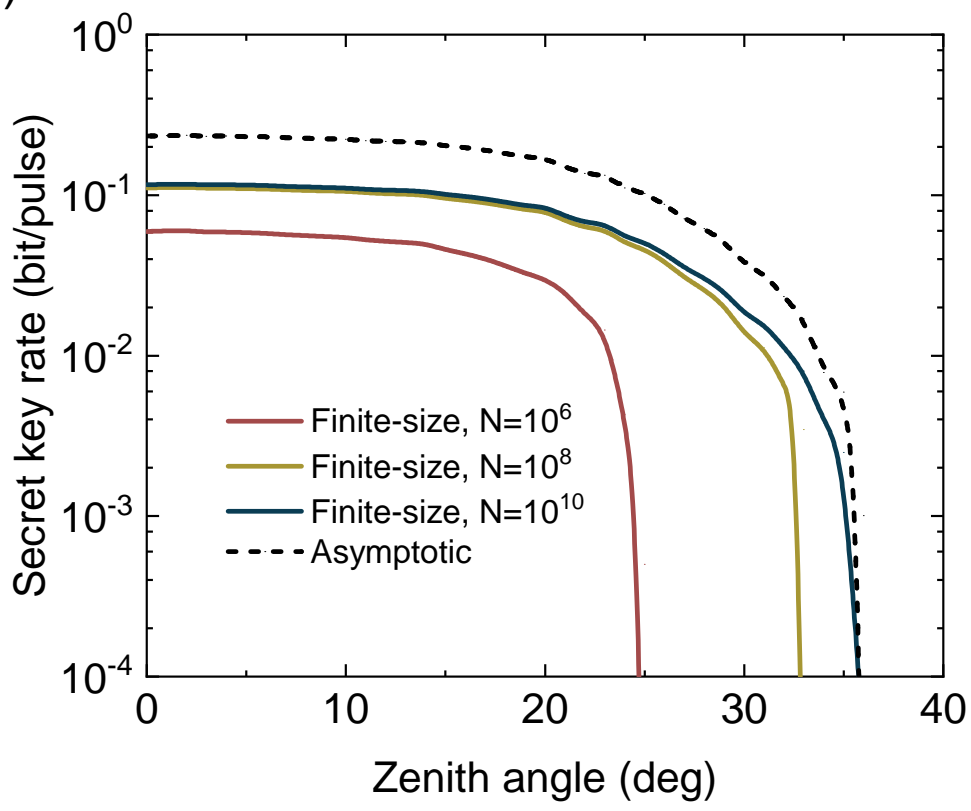

(b)

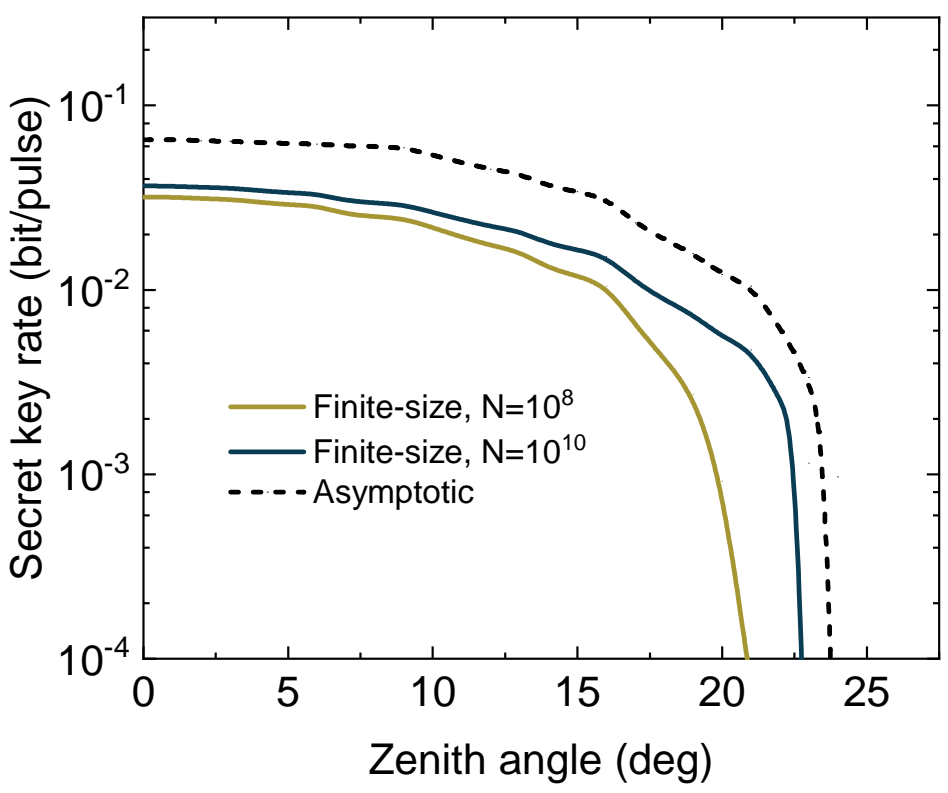

Figure 8. Secret key rate vs. zenith angle with finite-size effect for the satellite altitudes: (a) $H=100 \mathrm{~km}$; (b) $H=120 \mathrm{~km}$.

\section{Conclusions}

We have proposed an NA-based scheme for improving the uplink CV-QKD protocol. Specifically, the NA process is realized by ZPC operation, which happens to be a zeroenergy-input attenuation that does not cause the consumption of external energy. We find that this scheme shows better performance when the zenith angle is above $26^{\circ}$ and $3^{\circ}$ for $100 \mathrm{~km}$ and $120 \mathrm{~km}$, respectively. Numerical simulations show that the ZPC operation improves the performance by relatively reducing the eavesdropper's information. In addition, the ZPC-involved scheme performs better than the scheme with SPC operation, which is considered as another NA process. The research confirms the role of the NA operation on the uplink CV-QKD protocol and is conducive to making the CV-QKD protocol more practical. The limit of the proposed approach is that the effective time within 
a single communication cycle is still below $30 \mathrm{~s}$. The next step of our work is to reach a longer effective time.

Author Contributions: Conceptualization, Y.W. and Y.G.; methodology, Z.Z.; validation, S.X., Y.M. and Z.Z.; formal analysis, Y.M.; investigation, Y.L.; writing—original draft preparation, S.X.; writingreview and editing, Y.L.; visualization, X.R.; supervision, Y.G. All authors have read and agreed to the published version of the manuscript.

Funding: This work was funded by the National Natural Science Foundation of China (Grant No. 61871407), and Natural Science Foundation of Hunan Province, China (Grant No. 2021JJ30878).

Conflicts of Interest: The authors declare no conflict of interest.

\section{Abbreviations}

The following abbreviations are used in this manuscript:

QKD Quantum key distribution

DV Discrete-variable

CV Continuous-variable

NA Noiseless attenuation

ZPC Zero-photon catalysis

EB Entanglement-based

TMSV Two-mode squeezed vacuum

BS Beam splitter

PDT Probability distribution of transmittance

ABS Asymmetrical beam splitter

PNRD Photon number resolving detector

CF Characteristic function

Appendix A. The Elements of $\mu$ and $\Sigma$ in Equation (4)

The vector $\mu$ of Equation (4) has the form

$$
\mu=\left(\left\langle x_{0}\right\rangle,\left\langle y_{0}\right\rangle,\left\langle W_{1}^{2}\right\rangle,\left\langle W_{2}^{2}\right\rangle\right),
$$

where the elements $\left\langle x_{0}\right\rangle=\left\langle y_{0}\right\rangle=0$. The covariance matrix $\Sigma$ can be read as

$$
\Sigma=\left[\begin{array}{cccc}
\left\langle x_{0}^{2}\right\rangle & 0 & 0 & 0 \\
0 & \left\langle y_{0}^{2}\right\rangle & 0 & 0 \\
0 & 0 & \left\langle\Delta W_{1}^{2}\right\rangle & \left\langle\Delta W_{1} \Delta W_{2}\right\rangle \\
0 & 0 & \left\langle\Delta W_{1} \Delta W_{2}\right\rangle & \left\langle\Delta W_{2}^{2}\right\rangle
\end{array}\right]
$$

The details of $\mu$ and $\Sigma$ refer to the supplemental material of [29]. In this paper, we derive the $\mu$ and $\Sigma$ of uplink scenario with the equivalent ground-to-satellite atmospheric parameters in [17] together with the permittivity fluctuation spectrum [42]

$$
\Phi(\kappa)=0.132 C_{n}^{2}|\kappa|^{-\frac{11}{3}}+\frac{2 n_{0}}{\pi k^{4}}\left|f_{0}\left(\kappa ; d_{\text {scat }}\right)\right|^{2},
$$

where $k=2 \pi / \lambda_{0}$ is the wave number, $|\kappa| \in\left[\kappa_{0}, \kappa_{m}\right]$ with $\kappa_{0} \in 1 / L_{0}$ and $\kappa_{m} \in 1 / l_{0}\left(l_{0}\right.$ denotes the inner scales of turbulence). $f_{0}\left(\kappa ; d_{\text {scat }}\right)$ is the amplitude of the wave scattered from a separate particle, whose calculation method can be found in [43]. Note that we use the Mie scattering theory in this paper, as we consider the scattering from fog instead of rain [42]. The last two parameters of $\mu$ in the updated model can be calculated as

$$
\left\langle W_{i}^{2}\right\rangle=\frac{4 H^{2} \sec ^{2}(\zeta)}{k^{2} W_{0}^{2}}\left[\frac{\pi}{8} n_{0} W_{0}^{2} \bar{h} \sec (\zeta)+1.8 C_{n}^{2} k^{2} W_{0}^{\frac{5}{3}} \bar{h} \sec (\zeta)+1\right],
$$


where $\bar{h}=20 \mathrm{~km}$ denotes the altitude of the layer with prominent atmosphere effects. For covariance matrix $\Sigma$, the elements become

$$
\begin{array}{r}
\left\langle x_{0}^{2}\right\rangle=\left\langle y_{0}^{2}\right\rangle=1.16 \bar{h} C_{n}^{2} W_{0}^{-\frac{1}{3}} H^{2} \sec ^{3}(\zeta), \\
\left\langle\Delta W_{i} \Delta W_{j}\right\rangle=\left(2 \delta_{i j}-0.8\right) \frac{11 \bar{h} C_{n}^{2} H^{4} \sec ^{5}(\zeta)}{k^{2} W_{0}^{7}} \\
\times\left[1+\frac{\pi}{8} n_{0} W_{0}^{2} \bar{h} \sec (\zeta)\right] .
\end{array}
$$

More details of the downlink scenario based on the satellite-to-ground atmospheric parameters refer to our previous work [21].

Based on the elements above, we can estimate the probability distribution of transmittance (PDT) via the Monte Carlo method. In general, the transmittance $T$ can be estimated by

$$
T=T_{0} \exp \left\{-\left[\frac{d / a}{R\left(\frac{2}{W_{\text {eff }}(\varphi-\phi)}\right)}\right]^{Q\left(\frac{2}{W_{\text {eff }}(\varphi-\phi)}\right)}\right\},
$$

where $d=\sqrt{x_{0}^{2}+y_{0}^{2}}$ with the corresponding polar coordinates $(d, \phi), W_{\text {eff }}(\cdot)$ is the effective spot-radius with deformation effect, $T_{0}$ is the trancemiitance for the centered beam which can be calculated by

$$
\begin{array}{r}
T_{0}=1-I_{0}\left(a^{2}\left[\frac{1}{W_{1}^{2}}-\frac{1}{W_{2}^{2}}\right]\right) \exp \left[-a^{2}\left(\frac{1}{W_{1}^{2}}+\frac{1}{W_{2}^{2}}\right)\right] \\
-2\left\{1-\exp \left[-\frac{a^{2}}{2}\left(\frac{1}{W_{1}}-\frac{1}{W_{2}}\right)^{2}\right]\right\} \\
\quad \times \exp \left\{-\left[\frac{\frac{\left(W_{1}+W_{2}\right)^{2}}{\left|W_{1}^{2}-W_{2}^{2}\right|}}{R\left(\frac{1}{W_{1}}-\frac{1}{W_{2}}\right)}\right]^{\lambda\left(\frac{1}{W_{1}}-\frac{1}{W_{2}}\right)}\right\},
\end{array}
$$

where $R(\cdot)$ is the scale functions, $\lambda(\cdot)$ is the shape functions. The two functions can be expressed as

$$
\begin{gathered}
R(\xi)=\left[\ln \left(2 \frac{1-\exp \left[-\frac{1}{2} a^{2} \xi^{2}\right]}{1-\exp \left[-a^{2} \xi^{2}\right] I_{0}\left(a^{2} \xi^{2}\right)}\right)\right]^{-\frac{1}{\lambda(\xi)}}, \\
\lambda(\xi)=2 a^{2} \xi^{2} \frac{\exp \left(-a^{2} \xi^{2}\right) I_{1}\left(a^{2} \xi^{2}\right)}{1-\exp \left(-a^{2} \xi^{2}\right) I_{0}\left(a^{2} \tilde{\xi}^{2}\right)} \\
\times\left[\ln \left(2 \frac{1-\exp \left[-\frac{1}{2} a^{2} \tilde{\xi}^{2}\right]}{1-\exp \left[-a^{2} \tilde{\xi}^{2}\right] I_{0}\left(a^{2} \tilde{\xi}^{2}\right)}\right)\right]^{-1},
\end{gathered}
$$

where $I_{i}(\cdot)$ is the modified Bessel function of the $i$-th order.

\section{Appendix B. The Calculations of $I_{A B}$ and $\chi_{E}$}

The Shanon mutual information $I_{A B}$ can be explicitly obtained from the variances and the correlations between the modulation data and measurement data, which is given by [44]

$$
I_{A B}=\frac{1}{2} \log \frac{V_{A}}{V_{A \mid B}},
$$


where $V_{A \mid B}=V_{A}-C_{A B}^{2} / V_{B}$ is the conditional variance with the correlation $C_{A B}^{2}$ and measured data variance $V_{B}$. According to Equation (14), the mutual information between Alice and Bob in the homodyne detection case is given by

$$
I_{A B}=\frac{1}{2} \log _{2}\left(\frac{1}{1-\frac{\langle\sqrt{T}\rangle^{2} \gamma^{2}}{\langle T\rangle\left(X+\chi_{\text {line }}\right)(X+1)}}\right),
$$

where the factors $X=V$ and $Y=\sqrt{V^{2}-1}$ for coherent-state protocol.

The Holevo bound $\chi_{E}$ for reverse reconciliation is identified as [45]

$$
\chi_{E}=G\left(\frac{\lambda_{1}-1}{2}\right)+G\left(\frac{\lambda_{2}-1}{2}\right)-G\left(\frac{\lambda_{3}-1}{2}\right),
$$

where $G(X)=(x+1) \log _{2}(x+1)-x \log _{2} x, \lambda_{1,2,3}$ are the symplectic eigenvalues derived from the covariance matrices of the sharing quantum state [27]. In this paper, we consider the reverse reconciliation case so that $\chi_{E}$ denotes the information available to Eva on Bob's measurement results. According to Equation (14), the symplectic eigenvalues $\lambda_{1,2}$ are given by

$$
\lambda_{1,2}^{2}=\frac{\Delta \pm \sqrt{\Delta^{2}-4 D^{2}}}{2},
$$

with the notations

$$
\Delta=X^{2}+\langle T\rangle^{2}\left(X+\chi_{\text {line }}\right)^{2}-2\langle\sqrt{T}\rangle^{2} \gamma^{2},
$$

and

$$
D=\langle T\rangle X\left(X+\chi_{\text {line }}\right)-\langle\sqrt{T}\rangle^{2} \gamma^{2} .
$$

$\lambda_{3}$ in homodyne detection case is given by

$$
\lambda_{3}^{2}=X\left(X-\frac{\langle\sqrt{T}\rangle^{2} Y^{2}}{\langle T\rangle\left(X+\chi_{\text {line }}\right)}\right) .
$$

\section{Appendix C. Details of $\left\langle\beta_{a}^{2}\right\rangle$ in Uplink Case}

In uplink, the variance of $\beta_{a}$ follows

$$
\left\langle\beta_{a}^{2}\right\rangle \cong 2.91\left(\mu_{1 u}+0.62 \mu_{2 u} \Lambda^{11 / 6}\right) \sec (\zeta) \times(2 a)^{-1 / 3},
$$

where the factors $\mu_{1 u}$ and $\mu_{2 u}$ can be expressed as

$$
\begin{gathered}
\mu_{1 u}=\int_{0}^{\bar{h}} C_{n}^{2}\left(\Theta+\bar{\Theta} \cdot \frac{h}{H}\right)^{5 / 3} \mathrm{~d} h, \\
\mu_{2 u}=\int_{0}^{\bar{h}} C_{n}^{2}\left(1-\frac{h}{H}\right)^{5 / 3} \mathrm{~d} h .
\end{gathered}
$$

$\Lambda, \Theta$ and $\bar{\Theta}$ are the output-plane beam parameters, which describe the signal beam received at a distance $L=H \sec (\zeta)$ from the transmitter [32]. The output-plane beam parameters can be derived from the input-plane beam parameters, which is given by

$$
\begin{gathered}
\Theta_{0}=1-\frac{L}{F_{0}}, \\
\Lambda_{0}=\frac{2 L}{k W_{0}},
\end{gathered}
$$

where $F_{0}$ is the phase front radius of curvature at the transmitter output aperture. In this paper, we consider the collimated beam case so that the $F_{0}=\infty$. Then, the output-plane beam parameters can be expressed as 


$$
\begin{gathered}
\Theta=\frac{\Theta_{0}}{\Theta_{0}^{2}+\Lambda_{0}^{2}}=1+\frac{L}{F^{\prime}}, \\
\bar{\Theta}=1-\Theta, \\
\Lambda=\frac{\Lambda_{0}}{\Theta_{0}^{2}+\Lambda_{0}^{2}}=\frac{2 L}{k W^{2}},
\end{gathered}
$$

where $W$ denotes the diffractive beam radius at the receiver, and $F$ is the corresponding phase front radius of curvature.

\section{Appendix D. Derivation of $\gamma_{A B_{1}}$}

From [27], the coviriance matrix $\gamma_{A B_{1}}$ can be expressed as follows

$$
\gamma_{A B_{1}}=\left[\begin{array}{cccc}
\left\langle\hat{q}_{1}^{2}\right\rangle & 0 & \left\langle\hat{q}_{1} \hat{q}_{2}\right\rangle & 0 \\
0 & \left\langle\hat{p}_{1}^{2}\right\rangle & 0 & \left\langle\hat{p}_{1} \hat{p}_{2}\right\rangle \\
\left\langle\hat{q}_{1} \hat{q}_{2}\right\rangle & 0 & \left\langle\hat{q}_{2}^{2}\right\rangle & 0 \\
0 & \left\langle\hat{p}_{1} \hat{p}_{2}\right\rangle & 0 & \left\langle\hat{p}_{2}^{2}\right\rangle
\end{array}\right] .
$$

We need to calculate the six mean values in Equation (A26). Here we first introduce the input-output relation based on the characteristic function (CF). Then, the covariance matrix can be derived by using the relation between the mean values of observables and the $\mathrm{CF}$. First of all, the CF of TMSV is given by [46]

$$
\chi_{\text {in }}(\alpha, \beta)=e^{-\frac{V}{2}\left(|\alpha|^{2}+|\beta|^{2}\right)+\frac{\sqrt{V^{2}-1}}{2}\left(\alpha \beta+\alpha^{*} \beta^{*}\right)},
$$

The input-output relation of the ZPC operation can be theoretically expressed as

$$
\rho_{\text {out }, b}=\operatorname{Tr}_{b^{\prime}}\left[B \rho_{\text {in }, b}|0\rangle_{b^{\prime} b^{\prime}}\left\langle 0\left|B^{\dagger}\right| 0\right\rangle_{b^{\prime} b^{\prime}}\langle 0|\right],
$$

where B denotes the beam splitter operator. Therefore, the CF corresponding to $\rho_{\mathrm{out}, b}$ has the form

$$
\chi_{\text {out }}(\beta)=\int \frac{\mathrm{d}^{2} \gamma}{\pi R} \chi_{\text {in }}(\gamma) \chi_{0}\left(\gamma_{1}\right) \chi_{0}\left(\gamma_{2}\right),
$$

where $\gamma_{1}=\beta / \sqrt{R}-\gamma \sqrt{T_{A} / R}, \gamma_{2}=\gamma / \sqrt{R}-\beta \sqrt{T_{A} / R}, \chi_{\text {in }}$ is the CF of the input state, $\chi_{0}$ is the CF of the zero-photon Fock state with

$$
\chi_{0}\left(\gamma_{1}\right)=e^{-\frac{1}{2}\left|r_{1}\right|^{2}} L_{0}\left(\left|\gamma_{1}\right|^{2}\right),
$$

where $L_{0}(x)$ are the Laguerre polynomials. Here we use the Weyl expansion of the density operator, which has the form

$$
\rho=\int_{-\infty}^{\infty} \frac{\mathrm{d}^{2} \gamma}{\pi} \chi(\gamma) D(-\gamma)
$$

where $D(\gamma)$ is the displaced operator. Finally, the CF after the ZPC operation becomes

$$
\chi(\alpha, \beta)=\frac{1}{F_{0,0}(0,0)} \chi_{\mathrm{in}, \bar{\lambda}}(\alpha, \beta) F_{0,0}(\alpha, \beta),
$$

where $\chi_{\mathrm{in}, \bar{\lambda}}(\alpha, \beta)$ is the CF of the TMSV with a modified squeezing parameter $\bar{\lambda}=\lambda \sqrt{T}$, $F_{0,0}(\alpha, \beta)$ is the non-Gaussian term resulting from non-Gaussian operation given by

$$
F_{0,0}(\alpha, \beta)=\left(1-\lambda^{2}\right) /\left(1-\bar{\lambda}^{2}\right) .
$$


In terms of the relation between the mean values of observables and the CF [47], the mean values in Equation (A26) can be calculated as

$$
\begin{gathered}
\left\langle\hat{q}_{1}^{2}\right\rangle=-\left.\frac{\partial^{2}}{\partial p_{1}^{2}} \chi\left(0, p_{1} ; 0,0\right)\right|_{p_{1}=0,} \\
\left\langle\hat{p}_{1}^{2}\right\rangle=-\left.\frac{\partial^{2}}{\partial q_{1}^{2}} \chi\left(q_{1}, 0 ; 0,0\right)\right|_{q_{1}=0,} \\
\left\langle\hat{q}_{1} \hat{q}_{2}\right\rangle=-\left.\frac{\partial^{2}}{\partial p_{1} \partial p_{2}} \chi\left(0, p_{1} ; 0, p_{2}\right)\right|_{p_{1}, p_{2}=0,} \\
\left\langle\hat{p}_{1} \hat{p}_{2}\right\rangle=-\left.\frac{\partial^{2}}{\partial q_{1} \partial q_{2}} \chi\left(q_{1}, 0 ; q_{2}, 0\right)\right|_{q_{1}, q_{2}=0},
\end{gathered}
$$

where $\chi(\alpha, \beta)=\chi\left(q_{1}, p_{1} ; q_{2}, p_{2}\right)$ with $\alpha=q_{1}+i p_{1}$ and $\beta=q_{2}+i p_{2}$.

\section{References}

1. Luo, Y.H.; Chen, M.-C.; Erhard, M.; Zhong, H.; Wu, D. Quantum teleportation of physical qubits into logical code spaces. Proc. Natl. Acad. Sci. USA 2021, 118, 1-5. [CrossRef]

2. Ahmed, A.H.M.; Zakaria, M.N.; Metwally, N. Teleportation in the presence of technical defects in transmission stations. Appl. Math. Inf. Sci. 2012, 6, 781.

3. Pirandola, S.; Andersen, U.L.; Banchi, L.; Berta, M.; Bunandar, D.; Colbeck, M.; Englund, D.; Gehring, T.; Lupo, C.; Ottaviani, C.; et al. Advances in quantum cryptography. Adv. Opt. Photonics 2020, 12, 1012-1236. [CrossRef]

4. Hillery, M.; Bužek, V.; Berthiaume, A.; Gisin, N. Quantum secret sharing. Phys. Rev. A 1999, 59, 1829. [CrossRef]

5. Wu, X.; Wang, Y.; Huang, D. Passive continuous-variable quantum secret sharing using a thermal source. Phys. Rev. A 2020, 101, 022301. [CrossRef]

6. Liao, Q.; Liu, H.; Zhu, L.; Guo, Y. Quantum secret sharing using discretely modulated coherent states. Phys. Rev. A 2021, 103, 032410. [CrossRef]

7. Ahmed, A.M.; Cheong, L.Y.; Zakaria, N.; Metwally, N. Dynamics of Information Coded in a Single Cooper Pair Box. Int. J. Theor. Phys. 2013, 52, 1979-1988. [CrossRef]

8. Sridevi, R.; Philominathan, P.; Quantum Colour Image Encryption Algorithm Based on DNA and Unified Logistic Tent Map. Inf. Sci. Lett. 2020, 9, 9.

9. Ekert, A.K. Quantum cryptography based on Bell's theorem. Phys. Rev. Lett. 1991, 67, 661. [CrossRef]

10. Grosshans, F.; Grangier, P. Continuous variable quantum cryptography using coherent states. Phys. Rev. Lett. 2002, 88, 057902. [CrossRef] [PubMed]

11. Scarani, V.; Acin, A.; Ribordy, G.; Gisin, N. Quantum cryptography protocols robust against photon number splitting attacks for weak laser pulse implementations. Phys. Rev. Lett. 2004, 92, 057901. [CrossRef] [PubMed]

12. Jouguet, P.; Kunz-Jacques, S.; Leverrier, A.; Grangier, P.; Diamanti, E. Experimental demonstration of long-distance continuousvariable quantum key distribution. Nat. Photon. 2013, 7, 378-381. [CrossRef]

13. Xu, F.; Ma, X.; Zhang, Q.; Lo, H.K.; Pan, J.W. Secure quantum key distribution with realistic devices. Rev. Mod. Phys. 2020, 92, 025002. [CrossRef]

14. Liao, Q.; Xiao, G.; Zhong, H.; Guo, Y. Multi-label learning for improving discretely-modulated continuous-variable quantum key distribution. New J. Phys. 2020, 22, 083086. [CrossRef]

15. Wootters, W.K.; Zurek, W.H. A single quantum cannot be cloned. Nature 1982, 299, 802-803. [CrossRef]

16. Sidhu, J.S.; Siddarth, K.J.; Mustafa, G.; Thomas, B.; David, L.; Luca, M.; Markus, K.; Sonali, M.; Daniele, D.; Giuseppe, V.; et al. Advances in Space Quantum Communications. arXiv 2021, arXiv:2103.12749.

17. Liorni, C.; Kampermann, H.; Bruß, D. Satellite-based links for quantum key distribution: Beam effects and weather dependence. New J. Phys. 2019, 21, 093055. [CrossRef]

18. Vasylyev, D.; Vogel, W.; Moll, F. Satellite-mediated quantum atmospheric links. Phys. Rev. A 2019, 99, 053830. [CrossRef]

19. Bourgoin, J.P.; Meyer-Scot, E.; Higgins, B.L.; Erven, C.; Hube, H.; Kumar, H.; Hudson, D.; D'Souza, I.; Girard, R.; Laflamme, R.; et al. A comprehensive design and performance analysis of low Earth orbit satellite quantum communication. New J. Phys. 2013, 15, 023006. [CrossRef]

20. Pirandola, S. Satellite quantum communications: Fundamental bounds and practical security. Phys. Rev. Res. 2021, 3, 023130. [CrossRef]

21. Zuo, Z.; Wang, Y.; Huang, D.; Guo, Y. Atmospheric effects on satellite-mediated continuous-variable quantum key distribution. J. Phys. A Math. Theor. 2020, 53, 465302. [CrossRef]

22. Mičuda, M.; Straka, I.; Miková, M.; Dušek, M.; Cerf, N.J.; Fiurášek, J.; Ježek, M. Noiseless loss suppression in quantum optical communication. Phys. Rev. Lett. 2012, 109, 180503. [CrossRef] 
23. Fiurášek, J.; Cerf, N.J. Gaussian postselection and virtual noiseless amplification in continuous-variable quantum key distribution. Phys. Rev. A 2012, 86, 060302. [CrossRef]

24. Meng, G.; Yang, S.; Zou, X.; Zhang, S.; Shi, B.; Guo, G. Noiseless suppression of losses in optical quantum communication with conventional on-off photon detectors. Phys. Rev. A 2012, 86, 042305. [CrossRef]

25. Blandino, R.; Walk, N.; Lund, A.P.; Ralph, T.C. Channel purification via continuous-variable quantum teleportation with Gaussian postselection. Phys. Rev. A 2016, 93, 012326. [CrossRef]

26. Zhang, S.; Zhang, X. Photon catalysis acting as noiseless linear amplification and its application in coherence enhancement. Phys. Rev. A 2018, 97, 043830. [CrossRef]

27. Weedbrook, C.; Pirandola, S.; García-Patrón, R.; Cerf, N.J.; Ralph, T.C.; Shapiro, J.H.; Lloyd, S. Gaussian quantum information. Rev. Mod. Phys. 2012, 84, 621. [CrossRef]

28. Usenko, V.C.; Heim, B.; Peuntinger, C.; Wittmann, C.; Marquardt, C.; Leuchs, G.; Filip, R. Entanglement of Gaussian states and the applicability to quantum key distribution over fading channels. New J. Phys. 2012, 14, 093048. [CrossRef]

29. Vasylyev, D.; Semenov, A.A.; Vogel, W. Atmospheric quantum channels with weak and strong turbulence. Phys. Rev. Lett. 2016, 117, 090501. [CrossRef]

30. Zuo, Z.; Wang, Y.; Mao, Y.; Ruan, X.; Guo, Y. Security of quantum communications in oceanic turbulence. Phys. Rev. A 2021, 104, 052613. [CrossRef]

31. Grosshans, F.; Van Assche, G.; Wenger, J.; Brouri, R.; Cerf, N.J.; Grangier, P. Quantum key distribution using gaussian-modulated coherent states. Nature 2003, 421, 238-241. [CrossRef] [PubMed]

32. Andrews, L.C.; Phillips, R.L. Laser Beam Propagation through Random Media, 2nd ed.; SPIE Press: Bellingham, WA, USA, 2005; pp. 199-201.

33. Wang, S.; Huang, P.; Wang, T.; Zeng, G. Atmospheric effects on continuous-variable quantum key distribution. New J. Phys. 2018, 20, 083037. [CrossRef]

34. Zuo, Z.; Wang, Y.; Mao, Y.; Ye, W.; Hu, L.; Huang, D.; Guo, Y. Quantum catalysis-assisted attenuation for improving free-space continuous-variable quantum key distribution. J. Phys. B At. Mol. Opt. Phys. 2020, 53, 185501. [CrossRef]

35. Qi, B.; Zhu, W.; Qian, L.; Lo, H.K. Feasibility of quantum key distribution through a dense wavelength division multiplexing network. New J. Phys. 2010, 12, 103042. [CrossRef]

36. Wang, S.; Huang, P.; Wang, T.; Zeng, G. Feasibility of All-Day Quantum Communication with Coherent Detection. Phys. Rev. Appl. 2019, 12, 024041. [CrossRef]

37. Zuo, Z.; Wang, Y.; Liao, Q.; Guo, Y. Overcoming the uplink limit of satellite-based quantum communication with deterministic quantum teleportation. Phys. Rev. A 2021, 104, 022615. [CrossRef]

38. Leverrier, A.; Grosshans, F.; Grangier, P. Finite-size analysis of a continuous-variable quantum key distribution. Phys. Rev. A 2010, 81, 062343. [CrossRef]

39. Pirandola, S. Limits and security of free-space quantum communications. Phys. Rev. Res. 2021, 3, 013279. [CrossRef]

40. Liao, Q.; Xiao, G.; Xu, C.G.; Xu, Y.; Guo, Y. Discretely modulated continuous-variable quantum key distribution with an untrusted entanglement source. Phys. Rev. A 2020, 102, 032604. [CrossRef]

41. Ruppert, L.; Peuntinger, C.; Heim, B.; Günthner, K.; Usenko, V.C. Fading channel estimation for free-space continuous-variable secure quantum communication. New J. Phys. 2019, 21, 123036. [CrossRef]

42. Vasylyev, D.; Semenov, A.A.; Vogel, W.; Günthner, K.; Thurn, A.; Bayraktar, Ö.; Marquardt, C. Free-space quantum links under diverse weather conditions. Phys. Rev. A 2017, 96, 043856. [CrossRef]

43. Hulst, H.C.; van de Hulst, H.C. Light Scattering by Small Particles, 1st ed.; Dover: New York, NY, USA, 1981.

44. Xiang, Y.; Wang, Y.; Ruan, X.; Zuo, Z.; Guo, Y. Improving the discretely modulated underwater continuous-variable quantum key distribution with heralded hybrid linear amplifier. Phys. Scr. 2021, 96, 065103. [CrossRef]

45. Holevo, A.S.; Werner, R.F. Evaluating capacities of bosonic Gaussian channels. Phys. Rev. A 2001, 63, 032312. [CrossRef]

46. Hu, L.; Al-amri, M.; Liao, Z.; Zubairy, M.S. Continuous-variable quantum key distribution with non-Gaussian operations. Phys. Rev. A 2020, 102, 012608. [CrossRef]

47. Maniscalco, S.; Piilo, J.; Intravaia, F.; Petruccione, F.; Messina, A. Lindblad-and non-Lindblad-type dynamics of a quantum Brownian particle. Phys. Rev. A 2004, 70, 032113. [CrossRef] 